\title{
TRANSPORTE DE SOLUTOS NOSOLO E NO ESCOAMENTO SUPERFICIAL: I - DESENVOLVIMENTO DO MODELO E SIMULAÇÃO DO MOVIMENTO DE ÁGUA E ESCOAMENTO SUPERFICIAL ${ }^{1}$
}

\author{
Luiz Fernando C. de Oliveira ${ }^{2}$, Mauro Aparecido Martinez ${ }^{3}$, Fernando Falco Pruski ${ }^{4}$, \\ Hugo Alberto Ruiz ${ }^{5}$ \& Luiz Antônio Lima ${ }^{6}$

\section{RESUMO}

\begin{abstract}
Desenvolveu-se um modelo matemático para simulação do transporte de soluto no solo e no escoamento superficial. As equações diferenciais que regem os processos de transporte são resolvidas numericamente, pelo método das diferenças finitas. Para se avaliar o desempenho do modelo proposto, montou-se um experimento em nível de campo, constituído de nove parcelas, nas quais foram aplicadas três lâminas de irrigação com diferentes intensidades de precipitação; antes e após a aplicação da irrigação foram retiradas amostras de solo, para a obtenção dos perfis de umidade e, no final da parcela, coletou-se a vazão escoada superficialmente, pelo método direto. Os resultados simulados pelo modelo foram comparados com os experimentais, através do erro relativo médio. O modelo desenvolvido mostrou-se adequado para se descrever os processos de movimento de água no solo e escoamento superficial, apresentando comportamento semelhante aos das observações experimentais, podendo ser utilizado para simular esses processos, desde que os parâmetros de entrada do modelo sejam representativos.
\end{abstract}

Palavras-chave: transporte de solutos, escoamento superficial, transporte de sedimentos

\section{SOLUTE TRANSPORT IN SOIL AND SURFACE RUNOFF: I - MODEL DEVELOPMENT AND SIMULATION OF SOIL WATER MOVEMENT AND SURFACE RUNOFF}

\begin{abstract}
A mathematical model was developed to simulate solute transport in both soil and in surface runoff. The differential equations that govern the transport processes are numerically solved through the finite difference method. For the evaluation of the proposed model a field experiment was planned with nine plots under three irrigation levels with different rainfall intensities. Soil was sampled before and after irrigation to obtain moisture content profiles. At the end of the plot runoff flow was collected by the direct method. The model-simulated results were compared with the experimental data through the mean relative error. The developed model was found to describe adequately water movement and surface runoff, showing a behavior similar to experimental observations, making possible the utilization of the model to simulate these processes, if the model input parameters are representative.
\end{abstract}

Key words: solute transport, runoff, sediment transport

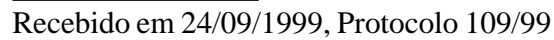

${ }^{1}$ Trabalho extraído da Tese de Doutorado do primeiro autor, em Engenharia Agrícola, UFV, parcialmente financiado pela FAPEMIG

${ }^{2}$ Professor da Escola de Agronomia, UFG, CEP 74001 - 970, Goiânia, GO. Fone: (0xx62) 821 1537, Fax: (0xx62) 205 1099.

E-mail: 1fco@agro.ufg.br

${ }^{3}$ Professor do Departamento de Engenharia Agrícola, UFV. Av. P.H. Rofs, s/n, CEP 36571 - 000, Viçosa, MG. Fone: (0xx31) 899 2730, Fax:(0xx31) 8992735.E-mail: mmauro@mail.ufv.br

${ }^{4}$ Professor do Departamento de Engenharia Agrícola, UFV. E-mail: ffpruski@mail.ufv.br

${ }^{5}$ Professor do Departamento de Solos, UFV. E-mail: hruiz@mail.ufv.br

${ }^{6}$ Professor do Departamento de Engenharia, UFLA. CEP 37200 - 000, Lavras, MG. E-mail: lalima@ibm.net 


\section{INTRODUÇÃO}

A atual intensificação do uso das terras para fins agrícolas tem despertado grande preocupação devido, principalmente, aos impactos que vem causando ao ambiente, sobretudo no que diz respeito à sua contaminação por substâncias químicas. Aplicados com o objetivo de aumentar a produtividade, esses produtos interagem com o solo e a água, por meio de diversos processos e, uma vez aplicados ao solo, podem ser transportados de dois modos: pela água da chuva ou da irrigação: sobre a superfície do solo, juntamente com a água de escoamento superficial, e através do perfil do solo com a água infiltrada (Piffer, 1989); assim, o transporte de solutos no solo constitui o principal veículo de contaminação das águas subterrâneas e superficiais.

A modelagem do movimento de água e solutos no solo é importante para a compreensão de formas para reduzir a poluição das águas superficiais e subterrâneas (Rogers, 1994). A possibilidade de se prever o movimento de solutos no solo e no escoamento superficial, por meio de modelos computacionais baseados na equação de transporte difusivo-convectivo, permite uma extraordinária economia de tempo e de recursos financeiros, os quais seriam gastos em estudos experimentais. Entretanto, a aplicação desses modelos de simulação para diversas condições precisa ser pesquisada, comparando-se os resultados simulados com aqueles observados em experimentos de campo (Clemente et al., 1993; Wallach \& Shabtai, 1993).

Segundo Govindaraju (1996) os modelos para simulação do transporte de solutos pelo escoamento superficial negligenciam a parcela de produto químico que, adsorvido às partículas do solo, é transportado como consequiência do processo erosivo. A inclusão desse processo físico resulta em melhoria na predição das perdas de solutos, pelo escoamento superficial.

O desenvolvimento de modelos matemáticos para se descrever, com precisão, o transporte de água e solutos, é bastante difícil. As equações que descrevem esses processos, em condições reais de campo, apresentam grande complexidade (Cox et al., 1994); no entanto, Kinouchi et al. (1991) e Wallach \& Shabtai (1992) consideram que a evolução dos recursos computacionais, aliada às técnicas numéricas, tem facilitado a modelagem do transporte de solutos, tornando possível integrar-se os múltiplos processos que determinam as mudanças na concentração dos solutos presentes no solo, no tempo e no espaço, o que permite melhor entendimento do comportamento dos processos associados ao transporte e à transformação dos solutos no solo. No Brasil, os poucos estudos realizados limitam-se à determinação do poder residual e à movimentação e degradação de pesticidas, de metais pesados, de nutrientes e de compostos orgânicos no solo.

Neste sentido, o presente trabalho teve como objetivo: desenvolver um modelo matemático capaz de predizer o transporte de solutos no solo e no escoamento superficial e simular os processos de movimento de água no solo e escoamento superficial.

\section{MATERIAL E MÉTODOS}

O modelo proposto neste trabalho simula os processos do movimento de água no solo, do transporte de solutos, escoamento superficial e transporte de sedimentos, pela utilização de equações diferenciais. As equações diferenciais são resolvidas numericamente, pelo método das diferenças finitas.

No desenvolvimento do modelo foi idealizada uma região de estudo, conforme Figura 1. Para se modelar o movimento de água e transporte de soluto no perfil do solo, considerou-se a superfície do solo como limite superior e a profundidade $\mathrm{z}_{\mathrm{p}}$ como limite inferior da região de estudo. $O$ comprimento da rampa " $x$ " foi delimitada à montante, pelo limite superior da faixa individualizada e, à jusante, pelo ponto de coleta do escoamento superficial.

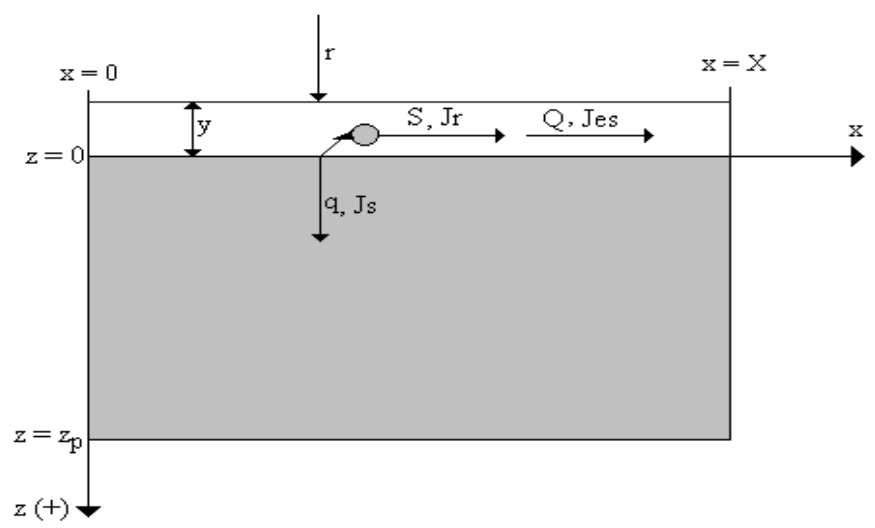

Figura 1. Região de estudo empregada no desenvolvimento do modelo

Na Figura 1, "r" representa a chuva ou irrigação, $\mathrm{L} \mathrm{T}^{-1} ; \mathrm{q}$ o fluxo de água no solo, $\mathrm{L} \mathrm{T}^{-1} ; \mathrm{y}$ a profundidade do escoamento superficial, L; Q a vazão escoada superficialmente, $\mathrm{L}^{3} \mathrm{~T}^{-1} ; \mathrm{S}$ os sedimentos transportados pelo escoamento superficial, $\mathrm{M} \mathrm{L}^{-2} \mathrm{~T}^{-1}$; $\mathrm{J}_{\mathrm{s}}$ o transporte de solutos no perfil do solo, $\mathrm{M} \mathrm{L} \mathrm{L}^{-2} \mathrm{~T}^{-1} ; \mathrm{J}_{\mathrm{es}} \mathrm{O}$ transporte de solutos em solução no escoamento superficial, $\mathrm{M}$ $\mathrm{L}^{-2} \mathrm{~T}^{-1} ; \mathrm{J}_{\mathrm{r}} \mathrm{o}$ transporte de solutos adsorvidos aos sedimentos no escoamento superficial, $\mathrm{ML}^{-2} \mathrm{~T}^{-1} ; \mathrm{z}_{\mathrm{p}}$ o limite inferior do perfil do solo, L; e X o comprimento da faixa, L.

$\mathrm{Na}$ análise do processo do movimento de água no solo foi utilizada a equação de Richards, considerando-se o escoamento unidimensional não saturado e a coordenada vertical, orientada positivamente para baixo, como segue:

$$
\mathrm{C}(\mathrm{h}) \frac{\partial \mathrm{h}}{\partial \mathrm{t}}=\frac{\partial}{\partial \mathrm{z}}\left[\mathrm{K}(\mathrm{h}) \frac{\partial}{\partial \mathrm{z}}(\mathrm{h}-\mathrm{z})\right]
$$

em que:

$\mathrm{C}(\mathrm{h}) \quad$ - capacidade hídrica específica do solo, $\mathrm{L}^{-1}$

h - potencial matricial, $\mathrm{L}$

$\mathrm{K}(\mathrm{h}) \quad$ - condutividade hidráulica do solo não saturado, $\mathrm{L} \mathrm{T}^{-1}$

z - coordenada vertical, $\mathrm{L}$

$\mathrm{t}$ - tempo, $\mathrm{T}$

As condições iniciais e de contorno utilizadas na resolução da Eq. 1, são:

$$
\begin{aligned}
& \text { - para } t=0 \text { e } 0 \leq \mathrm{z} \leq \mathrm{z}_{\mathrm{n}}, \theta_{\mathrm{i}}(\mathrm{z}, 0)=\left(\theta_{\mathrm{o}}\right)_{\mathrm{i}} \text { para } \mathrm{i}=1,2, \ldots, \mathrm{n} \\
& \text { - para } \mathrm{z}=0 \text { se vi } \geq \mathrm{r} \text { então } \mathrm{q}_{1}=\mathrm{r} \text { para } 0 \leq \mathrm{t} \leq \mathrm{t}_{\mathrm{p}} \\
& \text { - para } \mathrm{z}=0 \text { se vi }<\mathrm{r} \text { então } \mathrm{h}_{1}=0 \text { para } \mathrm{t}_{\mathrm{p}} \leq \mathrm{t} \\
& \text { - } \text { para } \mathrm{z}=\mathrm{z}_{\mathrm{p}} \text { e } 0 \leq \mathrm{t} \leq \mathrm{T},
\end{aligned}
$$

em que:

$\theta \quad$ - umidade volumétrica, $\mathrm{LL}^{-3}$ 
$t_{p}$ - tempo em que a velocidade de infiltração de água no solo (vi) se iguala à intensidade de precipitação (r), T

$\mathrm{q}_{1}$ - fluxo de água na superfície do solo, $\mathrm{L} \mathrm{T}^{-1}$

$\mathrm{h}_{\mathrm{p}}$ - potencial matricial do nó na profundidade $\mathrm{z}_{\mathrm{p}}, \mathrm{L}$

$\mathrm{T}$ - tempo total do evento (chuva ou irrigação)

n - número de nós da discretização do perfil do solo, T

Na obtenção dos parâmetros físico-hídricos do solo $\mathrm{C}(\mathrm{h}) \mathrm{e}$ $\mathrm{K}(\mathrm{h})$ o programa permite selecionar os modelos de van Genuchten (1980) e Brooks \& Corey (1964). Selecionada a opção, esses parâmetros são calculados pelo emprego das Eqs. 2 a 5, com base na distribuição do potencial de umidade no perfil do solo.

van Genuchten:

$$
\begin{gathered}
C(h)=\frac{m n \alpha^{\mathrm{n}}\left(\theta_{\mathrm{r}}-\theta_{\mathrm{s}}\right) \mathrm{h}^{\mathrm{n}-1}}{\left[1+(\alpha \mathrm{h})^{\mathrm{n}}\right]^{\mathrm{m}+1}} \\
\mathrm{~K}(\mathrm{~h})=\mathrm{K}_{\mathrm{o}}\left(\frac{\theta-\theta_{\mathrm{r}}}{\theta_{\mathrm{s}}-\theta_{\mathrm{r}}}\right)^{1 / 2}\left[1-\left(1-\left(\frac{\theta-\theta_{\mathrm{r}}}{\theta_{\mathrm{s}}-\theta_{\mathrm{r}}}\right)^{1 / \mathrm{m}}\right)^{\mathrm{m}}\right]^{2}
\end{gathered}
$$

Brooks \& Corey:

$$
\begin{gathered}
\mathrm{C}(\mathrm{h})=\frac{\lambda\left(\theta_{\mathrm{r}}-\theta_{\mathrm{s}}\right)}{\mathrm{h}}\left(\frac{\mathrm{h}_{\mathrm{b}}}{\mathrm{h}}\right)^{\lambda} \\
\mathrm{K}(\mathrm{h})=\mathrm{K}_{\mathrm{o}}\left(\frac{\mathrm{h}_{\mathrm{b}}}{\mathrm{h}}\right)^{2+3 \lambda}
\end{gathered}
$$

em que:

$\theta_{\mathrm{s}}$ e $\theta_{\mathrm{r}}$ - umidade volumétrica de saturação e residual, respectivamente, $\mathrm{L} \mathrm{L}^{-3}$

$\alpha \quad$ - parâmetro que depende do solo, $\mathrm{L}^{-1}$

$\mathrm{n}$ e $\mathrm{m}$ - parâmetros que dependem do solo, adimensional

$\mathrm{K}_{\mathrm{o}}$ - condutividade hidráulica do solo saturado, $\mathrm{L} \mathrm{T}^{-1}$

$h_{b} \quad$ - potencial matricial de entrada de ar, $\mathrm{L}$

$\lambda$ - expoente que depende da distribuição do tamanho de poros, adimensional

$\mathrm{Na}$ resolução da Eq. 1 o perfil do solo foi discretizado em compartimentos, a partir da superfície do solo, sendo a região de estudo delimitada pela superfície do solo e pela base do perfil, sendo o subescrito a posição do nó na malha de discretização em z e o superescrito o incremento de tempo (Figura 2).

Para simular o transporte de solutos no perfil do solo, empregou-se a equação abaixo (Wallach \& Shabtai, 1992):

$$
\frac{\partial}{\partial \mathrm{t}}\left(\theta \mathrm{C}+\rho_{\mathrm{g}} \mathrm{C}_{\mathrm{s}}\right)=\frac{\partial}{\partial \mathrm{z}}\left(\theta \mathrm{D} \frac{\partial \mathrm{C}}{\partial \mathrm{z}}-\mathrm{qC}\right)
$$

em que:

$$
\begin{array}{ll}
\mathrm{C} & \text { - concentração do soluto na solução do solo, } \mathrm{M} \mathrm{L}^{-3} \\
\rho_{\mathrm{g}} & \text { - massa específica do solo, } \mathrm{M} \mathrm{L}^{-3} \\
\mathrm{C}_{\mathrm{S}} & \text { - concentração do soluto adsorvido na matriz do solo, } \mathrm{M} \mathrm{M}^{-1} \\
\mathrm{D} & \text { - coeficiente de dispersão hidrodinâmica, } \mathrm{L}^{2} \mathrm{~T}^{-1} \\
\mathrm{q} & \text { - fluxo de água no solo, } \mathrm{L} \mathrm{T}^{-1}
\end{array}
$$

As condições inicial e de contorno empregadas na resolução da Eq. 2, foram:

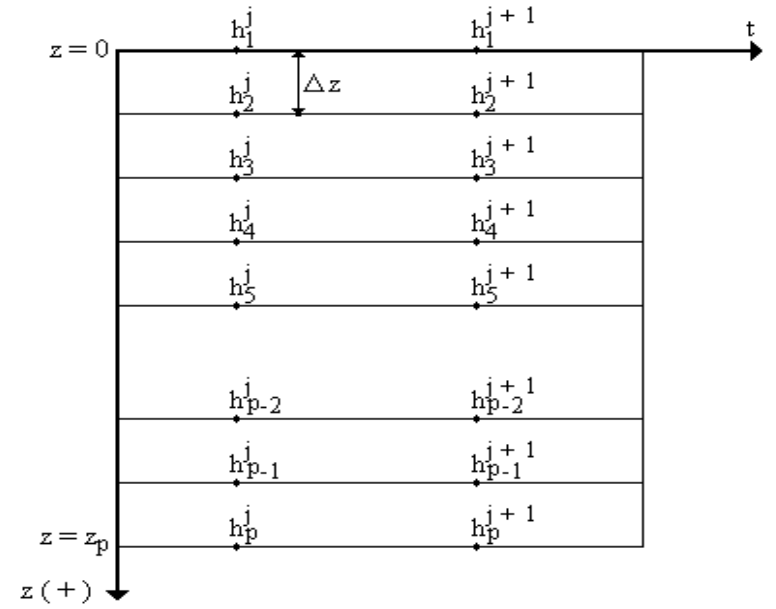

Figura 2. Discretização do perfil do solo em compartimentos

$$
\begin{aligned}
& \text { - para } \mathrm{t}=0 \text { e } 0 \leq \mathrm{z} \leq \mathrm{z}_{\mathrm{n}}, \mathrm{C}_{\mathrm{i}}(\mathrm{z}, 0)=\left(\mathrm{C}_{\mathrm{o}}\right)_{\mathrm{i}} \text { para } \mathrm{i}=1,2, \ldots, \mathrm{n} \\
& \text { - } \operatorname{para} 0 \leq \mathrm{t} \leq \mathrm{T} \text { e } \mathrm{z}=\mathrm{z}_{\mathrm{n}}, \mathrm{C}_{\mathrm{n}}^{\mathrm{j}}=\mathrm{C}_{\mathrm{p}} \\
& \text { - para } 0 \leq \mathrm{t} \leq \mathrm{t}_{\mathrm{p}} \text { e } \mathrm{z}=0, \mathrm{qC}-\theta \mathrm{D} \frac{\partial \mathrm{C}}{\partial \mathrm{z}}=-\mathrm{qC}_{\mathrm{a}}
\end{aligned}
$$

em que:

$\mathrm{C}_{\mathrm{a}}$ - concentração do soluto, na água da chuva ou irrigação, $\mathrm{ML}^{-3}$

$\mathrm{C}_{\mathrm{p}}$ - concentração do soluto do nó na profundidade zp, $\mathrm{M} \mathrm{L}^{-3}$

No momento em que a taxa de infiltração se torna menor que a intensidade de precipitação, inicia-se a formação de uma lâmina de água na superfície do solo. Por um período de tempo menor que o necessário para atender às abstrações iniciais $\left(\mathrm{t}_{\mathrm{IA}}\right)$, a concentração de solutos na lâmina formada na superfície do solo, foi considerada igual à concentração do nó posicionado nesta superfície. $\mathrm{O}$ escoamento superficial se iniciará quando as abstrações iniciais forem atendidas; no momento seguinte, a condição de contorno na superfície do solo será dada por:

$$
\text { - para } \mathrm{z}=0 \text { e } \mathrm{t}_{\mathrm{IA}} \leq \mathrm{t} \leq \mathrm{T},\left(\mathrm{qC}-\theta \mathrm{D} \frac{\partial \mathrm{C}}{\partial \mathrm{z}}\right)=-\Omega\left(\mathrm{C}_{(1, \mathrm{t})}-\mathrm{C}_{\mathrm{r}}\right)
$$

em que:

$\mathrm{C}_{(1, \mathrm{t})}$ - concentração do soluto na superfície do solo, $\mathrm{M} \mathrm{L}^{-3}$

$\Omega$ - coeficiente de transferência, $\mathrm{L} \mathrm{T}^{-1}$

$\mathrm{C}_{\mathrm{r}}$ - concentração do soluto em solução no escoamento superficial, $\mathrm{M} \mathrm{L}^{-3}$

Para o cálculo do tempo correspondente à ocorrência das abstrações iniciais $\left(\mathrm{t}_{\mathrm{IA}}\right)$ utilizou-se o método do número da curva, proposto pelo Soil Conservation Service (1972).

$\mathrm{Na}$ obtenção dos solutos e sedimentos transportados pelo escoamento superficial, o comprimento da rampa foi discretizado em células, conforme apresentado na Figura 3.

O escoamento superficial foi modelado utilizando-se a Eq. 3.

$$
\frac{\partial y}{\partial t}+\frac{\partial Q}{\partial x}=r-q
$$

em que:

Q - vazão por unidade de largura do canal, obtida pela equação de Manning, $\mathrm{L}^{2} \mathrm{~T}^{-1}$. As condições inicial e de contorno empregadas na resolução da Eq. 3, são:

- para $\mathrm{t}=\mathrm{t}_{\mathrm{IA}}$ e $0 \leq \mathrm{x} \leq \mathrm{L}, \mathrm{y}_{\mathrm{k}}=\mathrm{y}_{\mathrm{k}}{ }^{\mathrm{j}-1}$ para $\mathrm{k}=1$ a $\mathrm{M}$, $\mathrm{L}=$ comprimento da rampa, $\mathrm{x}=$ dimensão da célula e $\mathrm{M}=$ número 
de nós ao longo de $\mathrm{L}$

- para $\mathrm{t}_{\mathrm{IA}} \leq \mathrm{t} \leq \mathrm{T}$ e $\mathrm{x}=0, \mathrm{Q}_{\mathrm{k}-1}=0$, ou seja, não há contribuição de vazão das áreas à montante

- para $t_{I A} \leq t \leq T$ e $x=X, Q_{M+1}=Q_{M}$, ou seja, o nó $M+1$ se encontra posicionado dentro da calha coletora de vazão.

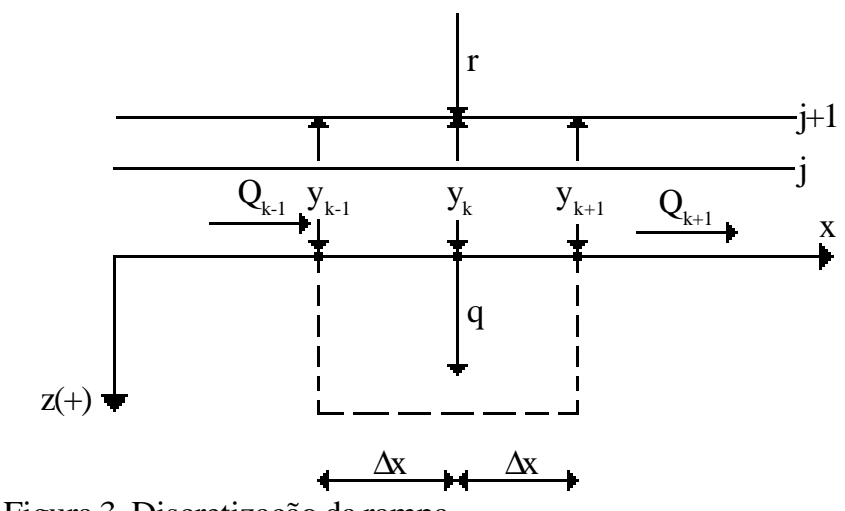

Figura 3. Discretização da rampa

O tempo considerado no modelo refere-se ao tempo de ocorrência do evento chuva; portanto, a fase do recesso não foi implementada.

Para simular o transporte de sedimentos, utilizou-se a Eq. 4.

$$
\frac{\partial \mathrm{S}}{\partial \mathrm{x}}+\frac{\partial(\mathrm{S} / \mathrm{v})}{\partial \mathrm{t}}=\mathrm{D}_{\mathrm{r}}+\mathrm{D}_{\mathrm{i}}
$$

em que:

S - quantidade de sedimentos transportada por unidade de largura e por unidade de tempo, $\mathrm{M} \mathrm{L}^{-1} \mathrm{~T}^{-1}$

$\mathrm{v} \quad$ - velocidade de escoamento, $\mathrm{L} \mathrm{T}^{-1}$

$\mathrm{D}_{\mathrm{i}}$ - erosão entre sulcos, $\mathrm{M} \mathrm{L}^{-2} \mathrm{~T}^{-1}$

$D_{\mathrm{r}} \quad$ - erosão em sulcos, $\mathrm{M} \mathrm{L}^{-2} \mathrm{~T}^{-1}$

Na resolução da Eq. 4, assumiram-se as seguintes condições inicial e de contorno:

- para $\mathrm{t}=\mathrm{t}_{\mathrm{IA}}$ e $0 \leq \mathrm{x} \leq \mathrm{L}, \mathrm{S}_{\mathrm{k}}^{\mathrm{j}}=\mathrm{S}_{\mathrm{k}+1}^{\mathrm{j}}$ e $\mathrm{D}_{\mathrm{i}_{\mathrm{k}+1}^{\mathrm{j}}}^{\mathrm{j}}=0$, para $\mathrm{k}=1 \mathrm{aM}$

- para $\mathrm{t}_{\mathrm{IA}} \leq \mathrm{t} \leq \mathrm{Te} \mathrm{x}=0, \mathrm{~S}_{\mathrm{k}}=0$, ou seja, não há contribuição de sedimentos das áreas à montante

- para $\mathrm{t}_{\mathrm{IA}} \leq \mathrm{t} \leq \mathrm{T}$ e $\mathrm{x}=\mathrm{L}, \mathrm{S}_{\mathrm{M}+1}=\mathrm{S}_{\mathrm{M}}$, ou seja, o nó $\mathrm{M}+1$ se encontra posicionado dentro da calha coletora de vazão.

A concentração dos solutos transportados em solução e adsorvidos aos sedimentos é obtida pelas equações:

$$
\begin{gathered}
\frac{\partial\left(\mathrm{y} \mathrm{C}_{\mathrm{r}}\right)}{\partial \mathrm{t}}=-(\mathrm{r}-\mathrm{q}) \mathrm{C}_{\mathrm{r}}+\Omega\left(\mathrm{C}-\mathrm{C}_{\mathrm{r}}\right) \\
\frac{\partial \mathrm{C}_{\mathrm{s}}}{\partial \mathrm{t}}=\frac{\partial}{\partial \mathrm{x}}\left(\mathrm{S} \mathrm{K}_{\mathrm{d}} \mathrm{C}\right)
\end{gathered}
$$

em que:

$\mathrm{K}_{\mathrm{d}}$ - coeficiente de partição.

Para se avaliar o desempenho do modelo proposto, montou-se um experimento em nível de campo, constituído de nove parcelas de $1 \mathrm{~m}$ de largura por $2 \mathrm{~m}$ de comprimento no sentido do declive. As parcelas foram delimitadas por chapas de flanders, com a finalidade de se individualizar o escoamento superficial. No final da parcela experimental instalou-se uma calha, com a finalidade de se recolher e conduzir o escoamento superficial para o ponto de coleta (Figura 4). Nas parcelas foram aplicadas três intensidades de precipitação 66,$6 ; 82,4$ e $100,4 \mathrm{~mm} \mathrm{~h}^{-1}$, durante uma hora. $\mathrm{O}$ volume de água escoado foi coletado pelo método direto, o que permitiu a obtenção das hidrógrafas de saída.

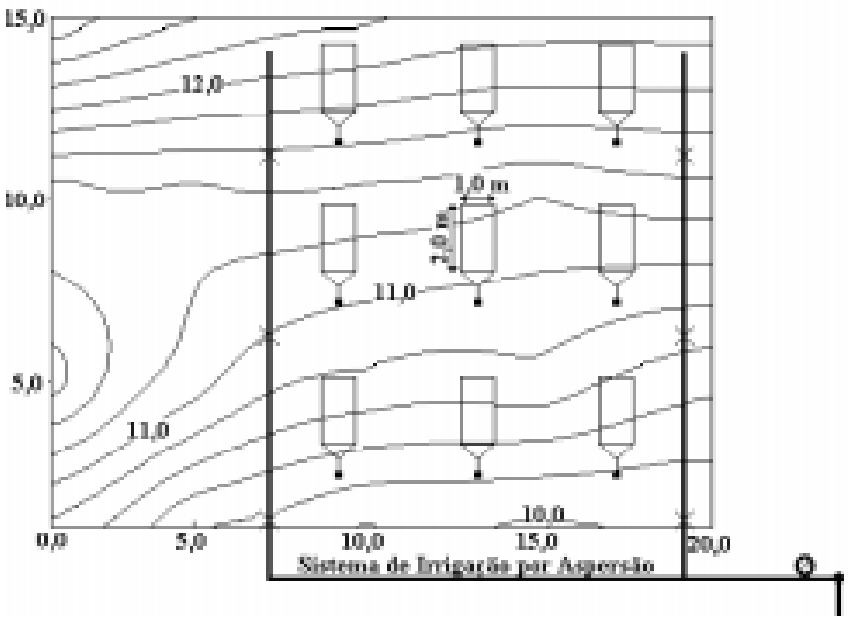

Figura 4. Esquema da área experimental

O solo da área experimental foi classificado como Podzólico Vermelho-Amarelo e o perfil dividido nos horizontes $A_{p}, A B$, $\mathrm{B}_{\mathrm{t}}, \mathrm{B}_{\mathrm{t} 2}, \mathrm{BC}$ e C, com profundidades de 0 a 10, 10 a 20,20 a 40, 40 a 60, 60 a 140, e de 140 a $250 \mathrm{~cm}$, respectivamente. Antes e após a aplicação das chuvas simuladas, foram retiradas amostras de solo nos diferentes horizontes, permitindo a obtenção dos perfis de umidade inicial e final.

Para se resolver numericamente as equações diferenciais empregadas no desenvolvimento do modelo, implementou-se um programa computacional em linguagem Delphi 3.0, denominado Modelo para Transporte de Solutos no Solo e no Escoamento Superficial - MTSES (Oliveira, 1999). Os resultados simulados pelo modelo foram comparados com os experimentais, pelo emprego do erro relativo médio (ERM).

$$
E R M=\frac{\sum_{\mathrm{i}=1}^{\mathrm{N}_{\text {OBS }}}\left|\frac{\mathrm{OBS}_{\mathrm{i}}-\mathrm{SIM}_{\mathrm{i}}}{\mathrm{OBS}_{\mathrm{i}}}\right|}{\mathrm{N}_{\mathrm{OBS}}}
$$

em que:

OBS e SIM - valores observados e estimados da variável de interesse, respectivamente

$\mathrm{N}_{\mathrm{OBS}}$ - número de observações

\section{RESULTADOS E DISCUSSÃO}

Com base nos parâmetros físico-hídricos do solo (Tabela 1), obtidos por Oliveira (1999), nos valores iniciais de umidade, simulou-se o perfil de umidade para as três intensidades de precipitação empregadas neste trabalho. Foram usados, nas simulações, incrementos de tempo $(\Delta t)$ de 30 s e de profundidade $(\Delta \mathrm{z})$ de $0,01 \mathrm{~m}$ e os modelos de van Genuchten (VG) e Brooks e Corey (BC). A espessura do perfil do solo utilizado nas simulações foi de $0,40 \mathrm{~m}$, uma vez que a frente de contaminação não atingiu o horizonte $\mathrm{B}_{\mathrm{t} 1}$.

Na Tabela 2 estão apresentados os valores dos erros relativos médios entre os perfis de umidade observados e os simulados, 
Tabela 1. Parâmetros físico-hídricos dos horizontes do Podzólico Vermelho-Amarelo

\begin{tabular}{ccccc}
\hline Horizonte Massa Específica & \begin{tabular}{c} 
Umidade \\
Solo Partículas \\
\cline { 2 - 3 } $\mathrm{kg} \mathrm{dm}^{-3}$
\end{tabular} & $\begin{array}{c}\text { Se Saturação } \\
\mathrm{m}^{3} \mathrm{~m}^{-3}\end{array}$ & $\begin{array}{c}\text { Condutividade Hidráulica } \\
\text { do Solo Saturado } \\
\mathrm{mm} \mathrm{h}^{-1}\end{array}$ \\
\hline $\mathrm{A}_{\mathrm{p}}$ & 1,23 & 2,59 & 0,523 & 2,330 \\
$\mathrm{AB}$ & 1,21 & 2,63 & 0,540 & 3,340 \\
$\mathrm{Bt}_{1}$ & 1,25 & 2,63 & 0,525 & 2,390 \\
$\mathrm{Bt}_{2}$ & 1,39 & 2,67 & 0,476 & 1,520 \\
$\mathrm{BC}$ & 1,42 & 2,71 & 0,485 & 1,990 \\
$\mathrm{C}$ & 1,39 & 2,70 & 0,479 & 1,700 \\
\hline
\end{tabular}

Tabela 2. Erros relativos médios (\%) para a simulação dos processos de movimento de água no solo e escoamento superficial, usando-se o Modelo para Transporte de Solutos no Solo e no Escoamento Superficial para os modelos de van Genuchten (VG) e Brooks e Corey (BC)

\begin{tabular}{lcrrr}
\hline Processos & \multicolumn{3}{c}{ Modelo Intensidades de Precipitação $\left(\mathrm{mm} \mathrm{h}^{-1}\right)$} \\
\cline { 3 - 5 } & & 66,6 & 82,4 & 100,4 \\
\hline Movimento de água no solo & VG & 2,7 & 3,3 & 4,4 \\
& BC & 2,7 & 3,6 & 4,7 \\
Escoamento superficial & VG & 9,8 & 13,6 & 21,2 \\
& BC & 10,8 & 14,2 & 22,4 \\
\hline
\end{tabular}

para as espessuras do perfil do solo de 0,40 m. De modo geral, os menores erros foram obtidos quando se utilizou, no MTSES, o modelo de van Genuchten.

$\mathrm{Na}$ Figura 5 tem-se os valores de umidade obtidos antes (inicial) e após (final) a aplicação das irrigações, e os perfis de umidade simulados para a espessura do perfil do solo de $0,40 \mathrm{~m}$ empregando-se, no MTSES, os modelos de van Genuchten e Brooks \& Corey. Pelo fato das intensidades de precipitação empregadas serem superiores à condutividade hidráulica do solo saturado para o horizonte $\mathrm{A}_{\mathrm{p}}$ e devido à curta duração da precipitação, a frente de umedecimento praticamente se manteve nas camadas superficiais do solo, como se pode observar na Figura 5. Embora a camada superficial do solo tenha atingido a saturação, a umidade referente ao ponto médio do horizonte $A_{p}$ foi menor que a de saturação. Para as três intensidades de precipitação utilizadas nas simulações, ambos os modelos, de Brooks \& Corey e van Genuchten, superestimaram o perfil de umidade para os primeiros $0,05 \mathrm{~m}$ do perfil do solo e, de modo geral, o modelo de Brooks \& Corey proporcionou maiores valores de umidade, quando comparado com o de van Genuchten. Essas variações são devidas ao fato de que o modelo de Brooks \& Corey geralmente superestima os valores da condutividade hidráulica, principalmente próximo à umidade de saturação, conforme constatado por Alexander \& Skaggs (1987), Martinez (1989) e Timm (1994). As diferenças entre os perfis de umidade observados e os simulados podem ser explicadas pelo fato do modelo permitir a obtenção dos valores de umidade para os nós empregados na discretização do perfil do solo, e a amostragem foi feita por horizonte do solo. Em cada horizonte, considerouse a umidade uniforme, sendo que, na realidade, este valor pode ser variável.

A vazão escoada superficialmente foi simulada para incremento do comprimento de rampa $\Delta \mathrm{x}=0,10 \mathrm{~m}$. Na simulação do escoamento superficial, utilizaram-se valores do coeficiente de Manning, variando entre 0,010 e 0,200, obtidos em Beasley \& Huggins (1981) com incrementos de 0,001. O valor do coeficiente de Manning, que resultou em menores valores dos erros relativos médios, foi de 0,019 para a condição de solo sem vegetação e preparo inicial, com ausência de restos vegetais.
(A) $100,4 \mathrm{~mm} \mathrm{~h}^{-1}$ Umidade $\left(\mathrm{m}^{3} \mathrm{~m}^{-3}\right)$

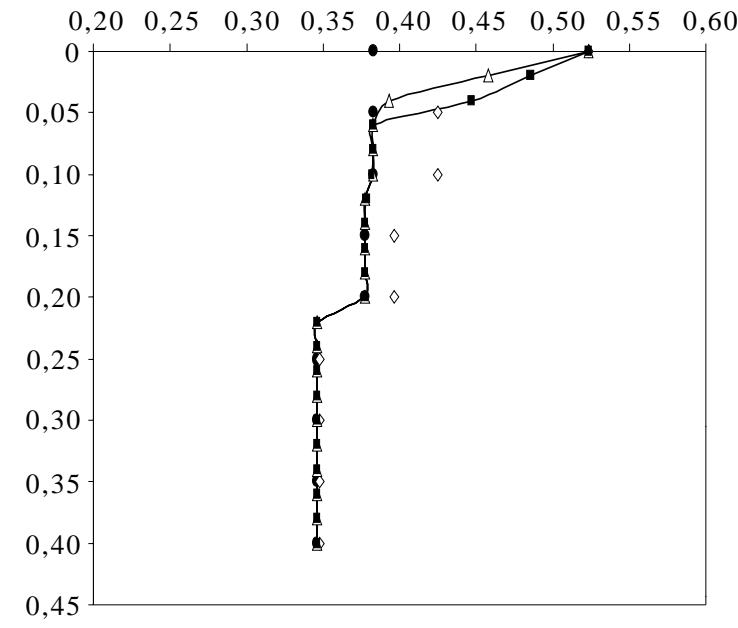

(B) $82,4 \mathrm{~mm} \mathrm{~h}^{-1}$

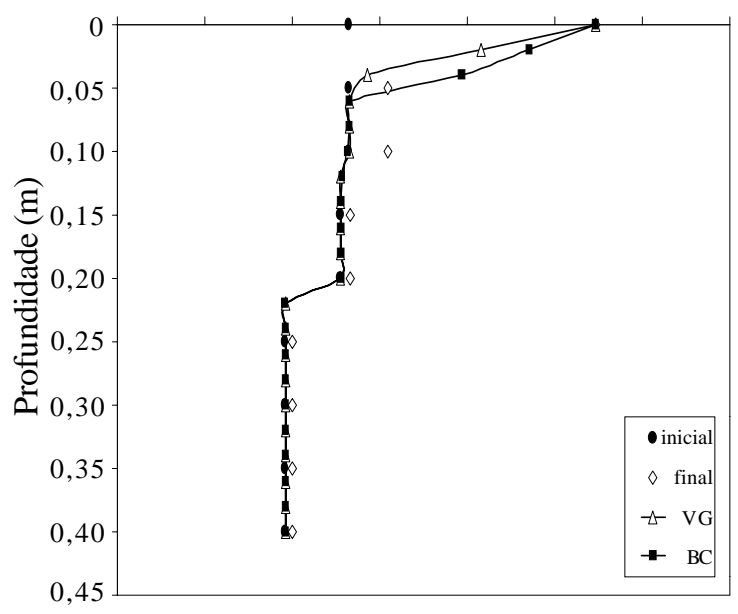

(C) $66,6 \mathrm{~mm} \mathrm{~h}^{-1}$

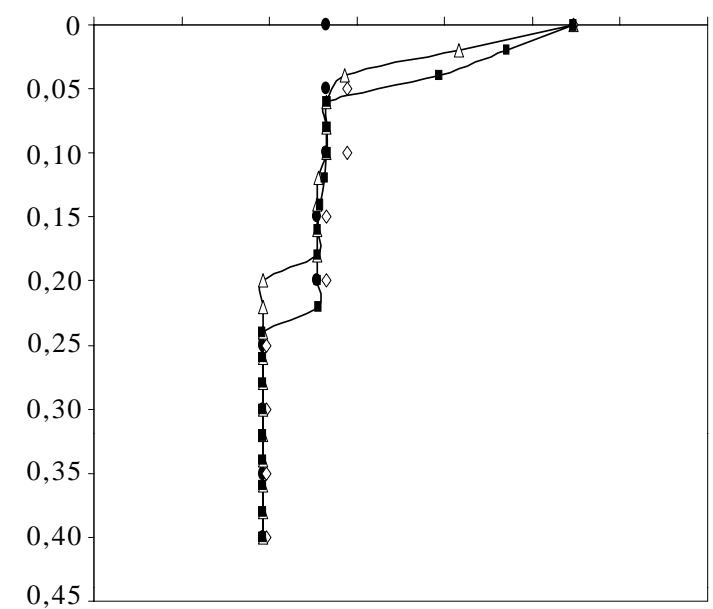

Figura 5. Umidade antes e após a irrigação e perfis de umidade simulados, empregando-se os modelos de van Genuchten (VG) e Brooks \& Corey (BC), para espessura do perfil do solo de $0,40 \mathrm{~m}, \Delta \mathrm{z}=0,01 \mathrm{~m}, \mathrm{e}$ intensidades de precipitação de (A) $100,4 \mathrm{~mm} \mathrm{~h}^{-1}$, (B) $82,4 \mathrm{~mm} \mathrm{~h}^{-1} \mathrm{e}$ (C) $66,6 \mathrm{~mm} \mathrm{~h}^{-1}$

Para o cálculo do tempo correspondente à ocorrência das abstrações iniciais, considerou-se uma condição média de umidade inicial do solo correspondente à definida pelo Soil 
Conservation Service, no método do número da curva, como AMC II, para o solo com superfície lavrada com sulcos retilíneos, com muito baixa capacidade de infiltração, gerando a maior proporção de escoamento superficial (tipo D) obtendo-se, assim, $\mathrm{CN}=94$ (Tucci, 1993).

Na Tabela 2 tem-se os valores dos erros relativos médios entre os valores de vazão observados e os simulados. Quando foi empregado o modelo de Brooks \& Corey, os valores dos ERM foram superiores aos obtidos pelo emprego do modelo de van Genuchten, cuja diferença se deve ao fato de que o modelo de Brooks \& Corey superestimou os valores de umidade ao longo do perfil do solo, acarretando a subestimativa dos valores das vazões.

O escoamento superficial iniciou-se aos 60, 240 e $250 \mathrm{~s}$ após o início das aplicações de água para as intensidades de 100,4; 82,4 e 66,6 $\mathrm{mm} \mathrm{h}^{-1}$, respectivamente. Os valores de vazão obtidos no experimento de campo, para as três intensidades de precipitação avaliadas, apresentaram comportamento crescente com o tempo, tendo em vista que a umidade dos diferentes horizontes se encontrava, inicialmente, com valores bem abaixo da umidade de saturação (Figura 6). Como a frente de umedecimento não atingiu grandes profundidades, a velocidade de infiltração e a vazão de saída não se estabilizaram. As hidrógrafas simuladas pelos modelos subestimaram os valores das vazões, embora tenham apresentado o mesmo comportamento das observações experimentais.

Na Tabela 3 estão apresentados os balanços de volume de água observado e simulado, para diferentes intensidades de precipitação, e o tempo correspondente à ocorrência das abstrações iniciais $\left(\mathrm{t}_{\mathrm{IA}}\right)$. Os volumes de água infiltrada no solo e escoada superficialmente, foram obtidos por integração numérica dos perfis de umidade e das hidrógrafas.

Para todas as intensidades de precipitação, os valores de $t_{I A}$ obtidos na simulação foram menores que os observados em nível de campo. Devido aos altos valores das intensidades de precipitação em relação à condutividade hidráulica do solo saturado da camada superficial, ocorreu a mudança na condição de contorno na simulação, o que fez com que o escoamento superficial se iniciasse antes do tempo observado experimentalmente.

Os volumes de água escoados e infiltrados obtidos com as simulações, aproximaram-se dos valores observados, confirmando os baixos valores dos erros relativos médios discutidos anteriormente. A baixa variabilidade dos valores de umidade obtidos experimentalmente e a metodologia empregada na determinação da vazão escoada, contribuíram para essa aproximação no balanço de volume. Os volumes de água escoados e computados pelo MTSES, quando se utilizou o modelo de Brooks \& Corey, foram menores que os obtidos quando se empregou o modelo de van Genuchten. Este comportamento pode ser explicado pelo fato do modelo de Brooks \& Corey haver superestimado o volume de água infiltrado no solo. De modo geral, os volumes de água infiltrada e escoada obtidos pela utilização do MTSES, foram super e subestimados com desvios em relação aos volumes
(A) $100,4 \mathrm{~mm} \mathrm{~h}^{-1}$

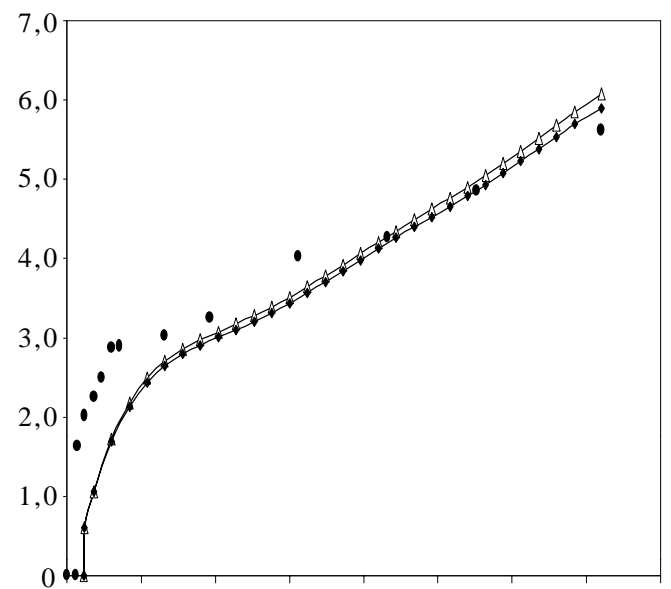

(B) $82,4 \mathrm{~mm} \mathrm{~h}^{-1}$

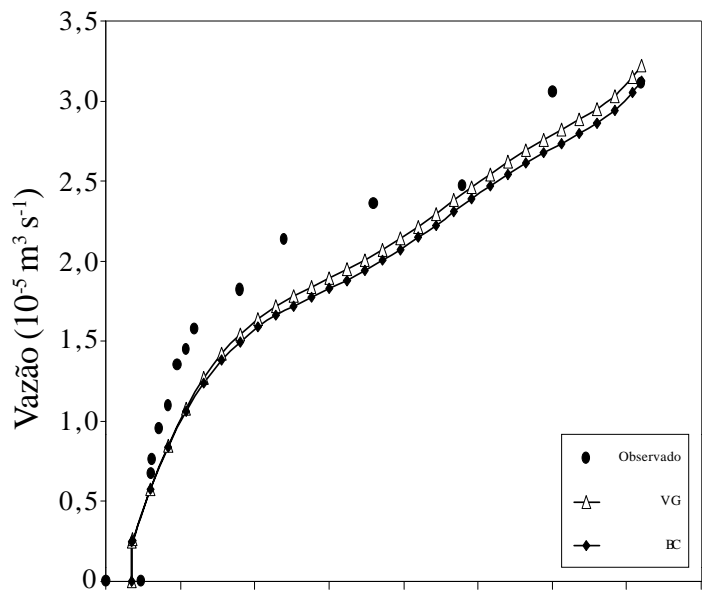

(C) $66,6 \mathrm{~mm} \mathrm{~h}^{-1}$

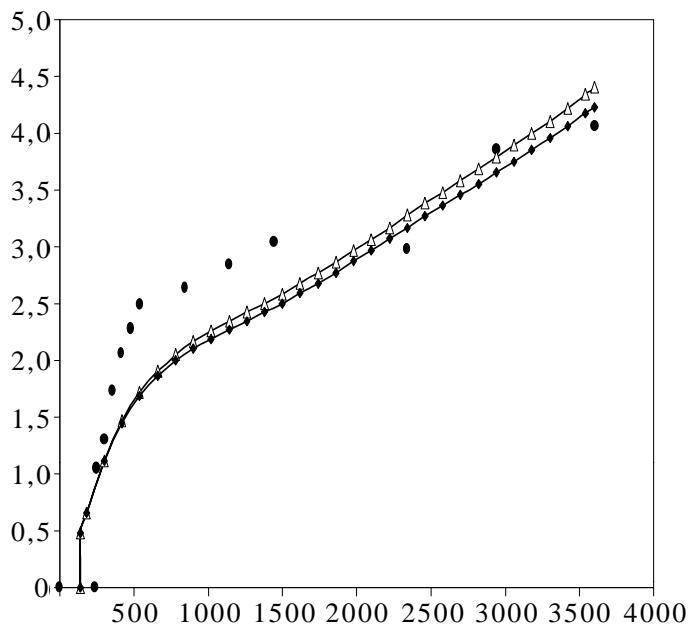

Tempo (s)

Figura 6. Vazões observadas e hidrógrafas simuladas para $\Delta \mathrm{x}=0,10 \mathrm{~m}$ e intensidades de precipitação de (A) $100,4 \mathrm{~mm} \mathrm{~h}^{-1}$, (B) $82,4 \mathrm{~mm} \mathrm{~h}^{-1} \mathrm{e}$ (C) $66,6 \mathrm{~mm} \mathrm{~h}^{-1}$

experimentais, variando de 0,2 a $25,0 \%$ e $-1,1$ a -10,7\%, respectivamente. O balanço volumétrico obtido pela utilização do MTSES foi subestimado quando se empregou o modelo de van Genuchten e superestimado quando se usou o modelo de Brooks \& Corey, com desvios variando de $-8,2$ a $2,7 \%$. 
Tabela 3. Balanço volumétrico e de massa observado (OBS) e simulado (SIM) pelo emprego dos modelos de van Genuchten $(V G)$ e Brooks \& Corey $(B C)$ para todas as intensidades de precipitação $\left(I_{p}\right)$ e tempo correspondente à ocorrência das abstrações iniciais

\begin{tabular}{|c|c|c|c|c|c|c|c|c|c|}
\hline \multirow[t]{2}{*}{$\begin{array}{c}\mathrm{I}_{\mathrm{p}} \\
\left(\mathrm{mm} \mathrm{h}^{-1}\right)\end{array}$} & \multicolumn{2}{|c|}{$\begin{array}{l}\mathrm{t}_{\mathrm{IA}} \\
(\mathrm{s})\end{array}$} & \multirow[t]{2}{*}{$\begin{array}{c}\text { Volume de Água } \\
\text { Aplicado }\left(\mathrm{m}^{3}\right)\end{array}$} & \multicolumn{3}{|c|}{$\begin{array}{l}\text { Volume de Água } \\
\text { Infiltrado }\left(\mathrm{m}^{3}\right)\end{array}$} & \multicolumn{3}{|c|}{$\begin{array}{l}\text { Volume de Água } \\
\text { Escoado }\left(\mathrm{m}^{3}\right)\end{array}$} \\
\hline & OBS & SIM & & OBS & $\mathrm{VG}$ & $\mathrm{BC}$ & OBS & $\mathrm{VG}$ & $\mathrm{BC}$ \\
\hline 82,4 & 240 & 141,7 & 0,1656 & 0,0566 & 0,0648 & 0,0707 & 0,1003 & 0,0992 & 0,0974 \\
\hline 100,4 & 60 & 116,3 & 0,2016 & 0,0593 & 0,0690 & 0,0737 & 0,1439 & 0,1335 & 0,1332 \\
\hline
\end{tabular}

\section{CONCLUSÕES}

1. O modelo desenvolvido mostrou-se adequado para descrever os processos de movimento de água no solo e escoamento superficial; estes, por sua vez, apresentaram comportamento semelhante ao das observações experimentais, podendo ser utilizados para simular tais processos, desde que os parâmetros de entrada do modelo sejam representativos.

2. De um modo geral, quando se empregou o modelo de Brooks \& Corey na obtenção da condutividade hidráulica do solo não-saturado e da capacidade hídrica do solo, os resultados das simulações superestimaram o volume de água infiltrada no solo e subestimaram o volume de água escoada, superficialmente, o mesmo não se verificou quando se empregou o modelo de van Genuchten.

\section{REFERÊNCIAS BIBLIOGRÁFICAS}

ALEXANDER, L.; SKAGGS, R.W. Predicting unsaturated hydraulic conductivity from the soil texture. Journal of Irrigation and Drainage Engineering, New York, v.113, n.2, p.184-197, 1987.

BEASLEY, D.B.; HUGGINS L.F. ANSWERS: User's manual. Chicago: United States Environmental Protection Agency, 1981.51p.

BROOKS, R.H.; COREY, A.T. Hydraulic properties of porous media. Colorado: Colorado State University, 1964. 27p. Hydrology Paper N3

CLEMENTE, R.S.; PRASHER, S.O.; BARRINGTON, S.F. PESTFADE, a new pesticide fate and transport model: Model development and verification. Transactions of the ASAE, St. Joseph, v.36, n.2, p.357-367, 1993.

COX, C.L.; JONES, W.F.; QUISENBERRY, V.L.; YO, F. One-dimensional infiltration with moving finite elements and improved soil water diffusivity. Water Resources Research, Washington, DC, v.30, n.5, p.1431-1438, 1994.
GOVINDARAJU, R.S. Modeling overland flow contamination by chemicals mixed in shallow soil horizons under variable source area hydrology. Water Resources Research, Washington, DC, v.32, n.3, p.753-758, 1996.

KINOUCHI, T.; KANDA, M.; HINO, M. Numerical simulation of infiltration and solute transport in S-shaped model basin by a boundary-fitted grid system. Journal of Hydrology, Amsterdam, v.122, n.1/4.p.373-406, 1991.

MARTINEZ, M.A. Modeling subsurface drainage in Clermont silt loam using finite element technique. West Lafeyete: Purdue University, 1989.173p. Doctor of Philosophy. Thesis OLIVEIRA, L.F.C. Modelo para transporte de solutos no solo e no escoamento superficial. Viçosa: UFV, 1999. 171p. Tese Doutorado

PIFFER, R. Movimento e degradação de Aldicarbe e Sulfona de Aldicarbe em dois diferentes solos. Lavras: ESAL, 1989. 99p. Dissertação Mestrado

ROGERS, J.S. Capacitance and initial time step effects on numerical solutions of Richards equation. Transactions of the ASAE, St. Joseph, v.37, n.3, p.807-813, 1994.

SOIL CONSERVATION SERVICE. National engineering handbook. s.1., 1972. n.p. Section 4: Hydrology

TIMM, L.C. Avaliação de alguns modelos matemáticos para a determinação da condutividade hidráulica de solos não saturados. Viçosa: UFV, 1994. 74p. Dissertação Mestrado

TUCCI, C.E.M. Escoamento superficial. In: TUCCI, C. E. M. Hidrologia, ciência e aplicação. Porto Alegre: ABRH, 1993. p.391-441.

van GENUCHTEN, M.T. A closed-form equation for predicting the hydraulic conductivity of unsaturated soils. Soil Science Society American Journal, Madison. v.44, p.892-898, 1980.

WALLACH, R.; SHABTAI, R. Modeling surface runoff contamination by chemicals under transient water infiltration. Journal of Hydrology, Amsterdam, v.132, n. 1/4, p.263-281, 1992.

WALLACH, R.; SHABTAI, R. Surface runoff contamination by chemicals initially incorporated below the soil surface. Water Resources Research, Washington, DC, v.29, n.3, p.697-704, 1993. 